\title{
Studies on Effects of Welding Parameters on the Mechanical Properties of Welded Low-Carbon Steel
}

\author{
M. A. Bodude ${ }^{*}$, I. Momohjimoh² \\ ${ }^{1}$ Department of Metallurgical and Materials Engineering, University of Lagos, Lagos, Nigeria \\ ${ }^{2}$ Department of Metallurgical Engineering, Yaba College of Technology, Lagos, Nigeria \\ Email: mbodude@unilag.edu.ng, momohjimoh ibrahim@yahoo.com
}

Received 25 February 2015; accepted 25 March 2015; published 31 March 2015

Copyright (C) 2015 by authors and Scientific Research Publishing Inc.

This work is licensed under the Creative Commons Attribution International License (CC BY). http://creativecommons.org/licenses/by/4.0/

(c) $\underset{\mathrm{EY}}{\mathrm{B}}$ Open Access

\begin{abstract}
In this work, the effect of heat input on the mechanical properties of low-carbon steel was studied using two welding processes: Oxy-Acetylene Welding (OAW) and Shielded Metal Arc Welding (SMAW). Two different edge preparations on a specific size, 10-mm thick low-carbon steel, with the following welding parameters: dual welding voltage of $100 \mathrm{~V}$ and $220 \mathrm{~V}$, various welding currents at 100, 120, and 150 Amperes and different mild steel electrode gauges of 10 and 12 were investigated. The tensile strength, hardness and impact strength of the welded joint were carried out and it was discovered that the tensile strength and hardness reduce with the increase in heat input into the weld. However, the impact strength of the weldment increases with the increase in heat input. Besides it was also discovered that $V$-grooved edge preparation has better mechanical properties as compared with straight edge preparation under the same conditions. Microstructural examinations conducted revealed that the cooling rate in different media has significant effect on the microstructure of the weldment. Pearlite and ferrite were observed in the microstructure, but the proportion of ferrite to pearlite varied under different conditions.
\end{abstract}

\section{Keywords}

Low Carbon Steel, Shielded Metal Arc Welding (SMAW), Oxy-Acetylene Welding (OAW), Heat Affected Zone (HAZ)

\section{Introduction}

Fusion welding processes are widely used for fabrications in many engineering applications such as aerospace,

"Corresponding author.

How to cite this paper: Bodude, M.A. and Momohjimoh, I. (2015) Studies on Effects of Welding Parameters on the Mechanical Properties of Welded Low-Carbon Steel. Journal of Minerals and Materials Characterization and Engineering, 3, 142-153. http://dx.doi.org/10.4236/immce.2015.33017 
automobile and ship building industries. Oxy-Acetylene Welding (OAW) is the most commonly used gas welding process because of its high flame temperature. Gas welding is a welding process that melts and joins the metals by heating them with flame caused by the reaction between a fuel gas and oxygen [1].

The main advantage of the OAW process is that the equipment is simple, portable and inexpensive. The total heat input per unit length of the weld in OAW process is rather high, resulting in large Heat Affected Zone (HAZ) which causes severe distortion [2]. Shielded Metal Arc Welding (SMAW) is a process that melts and joins metals by heating them with an arc established between a stick-like covered electrode and the metals. The welding equipment is also simple, portable and inexpensive [3].

One of the limitations of the two welding processes mentioned above as reported by Sindo [1] is the low power density of the heat source. The power density increases from a gas flame to an electric arc and a highenergy beam, and as the power density of the heat source increases, the heat input to the work piece that is required for welding decreases. The portion of the work piece material exposed to a gas flame heats up so slowly that before any melting occurs, a large amount of heat is already conducted away into the bulk of the work piece. Excessive heating can cause damage to the work piece, including weakening and distortion [4]. However, control of welding parameters such as welding current, voltage and speed can reduce the amount of heat input into the weld. Besides, edge preparation prior to welding can significantly affect the quality of the weld.

In view of the fact that arc welding processes like SMAW offer a wide spectrum of thermal energy for joining different thicknesses of steel, it was considered important that undertaking the present study would be very beneficial in gaining an understanding of the mechanical properties of low-carbon steel that influence the service performance of the welded joints under different heat input combinations i.e. low heat input and high heat input [5] [6].

The chemical composition of steel has impact on the weldability and the mechanical properties of the material. According to Monika et al. [7], several elements are purposefully added in the production of structural steel, but other undesirable elements may equally be present arising from the scrap materials charged during the steelmaking process. Carbon, manganese, tungsten and other elements increase strength and may increase the risk of cold cracking and therefore higher preheat and inter pass temperatures, better hydrogen control and sometimes post heat are necessary to avoid cracking [7].

Ueji [3] noted that weld fusion zone microstructure of low-carbon steel depends on the chemical composition of the material and the cooling rate. For steels, the required critical cooling rate for achieving martensite in the microstructure can be estimated using the following equation

$$
\log v=7.42-3.13 \mathrm{C}-0.71 \mathrm{Mn}-0.37 \mathrm{Ni}-0.34 \mathrm{Cr}-0.45 \mathrm{Mo}
$$

where $v$ is the critical cooling rate in $\mathrm{kmol} / \mathrm{hr}$.

Volume of melted metal is a function of heat input which in turn is governed by the welding parameters such as welding current and welding time. Increasing current decreases the fusion zone size while increasing time increases the fusion zone size [8] [9].

Ultrafine grained refinement from conventional grained size $(10 \mu \mathrm{m})$ to less than $1 \mu \mathrm{m}$ provides a preferable high strength to metallic materials used for structural applications as reported by Lowe and Zhu [10]. Ultrafine grained steel structural component in engineering application can be done by welding to produce complex structure which is difficult to manufacture directly. The major disadvantage of fusion welding of this ultrafine grained steel is the occurrence of grained growth during welding leading to reduction in strength [3]. Careful control of welding parameters can reduce the amount of heat input into the weld thereby avoiding the excessive grained growth and allotropic transformation in metals, thus improving the mechanical properties of the welded steel metal.

This work is therefore aimed at investigating the effects of welding current and voltage at two different edge preparations on the mechanical properties of low-carbon steel, using oxy-acetylene and shielded metal arc welding processes.

\section{Experimental}

\subsection{Materials}

Hot-rolled plate of low-carbon steel was obtained from a local metal market (Owode-Onirin) in Lagos, Nigeria. $10 \mathrm{~mm}$ thickness samples of the steel plate were cut and prepared for welding. E6013 electrode with gauge size 
10 and 12 were selected for the shielded metal arc welding (SMAW) process.

\subsection{Procedure}

Two edge preparations were done to perform welding on Butt Straight and single V groove joints. The test specimen was prepared in two $200 \times 50 \times 10 \mathrm{~mm}$ thick steel plates pieces and then welded together to give a finished test plate $200 \times 100 \times 10 \mathrm{~mm}$ with a weld down the middle as shown in Figure 1 . Three stages were followed for the experiment. In the first stage the metals plate with the prepared edges were taken for forming the welded joint. The material pairs selected for forming the welded joints are shown in Figure 1.

The second stage was the welding process and the joint formation using different process parameters.

The third stage was undertaken to study the effect of welding parameters on the mechanical properties and microstructure of welded joint.

The pieces of steel cut were paired and aligned on a table by the use of an angular Iron and then setting up of welding circuit before welding. Welding of samples in horizontal position was performed continuously by SMAW using the varied welding parameters: dual voltage device $100 \mathrm{~V}$ and $220 \mathrm{~V}$, various welding current at 100, 120 and 150 Amperes.

\subsection{Hardness Testing}

Hardness testing of welding joints was performed in accordance to ASTM A370-14 standard using Rockwell Testing Machine. The sample specimen was placed with the surface on the anvil, and slowly turning the hand wheel until the specimen was raised to touch the indenter. The numbers were read directly from the dial indicator and converted to the Rockwell number.

Hardness Test was done in a row to ensure that the Base Material, Heat Affected Zone and Weld Metal at a distance $\leq 2 \mathrm{~mm}$ from surface and $2 \mathrm{~mm}$ from fusion line were captured. Schematic representation of Butt and Groove welded joint with the locations of hardness testing and examination of microstructure is shown in Figure 2.

Rockwell Micro Hardness traverses (1-mm increments) were produced across the weld regions using a 150-g load and a $10 \mathrm{~s}$ dwell time.

\subsection{Tensile Testing}

Tensile Test was conducted on the welded steel sample using a hydraulic extensometer with prepared machined specimen dimensioned $85 \times 50 \times 10 \mathrm{~mm}$. The tensile testing was done in accordance to ASTM A370-14 standard. Tensile samples were prepared by milling of the top and bottom surfaces to remove flashing and other surface irregularities. The standard shape is obtained using lathe machine as shown in the Figure 3.

\subsection{Impact Testing}

Test specimens with a $\mathrm{V}$ notch in the fusion zone for impact test fracture were performed according to ASTM E23 standard.

The prepared specimen was placed on the Anvil with V-notch gauge. The Pendulum was set to predetermined level 220ft.Ib (298.642 J) and released to strike and fracture the positioned specimen. The value of Energy Impacted was taken and recorded.

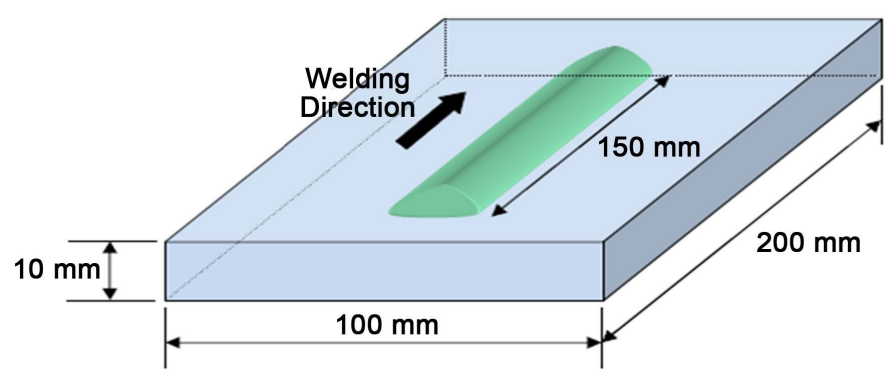

Figure 1. Schematic illustration of the bead on plate location and dimensions. 


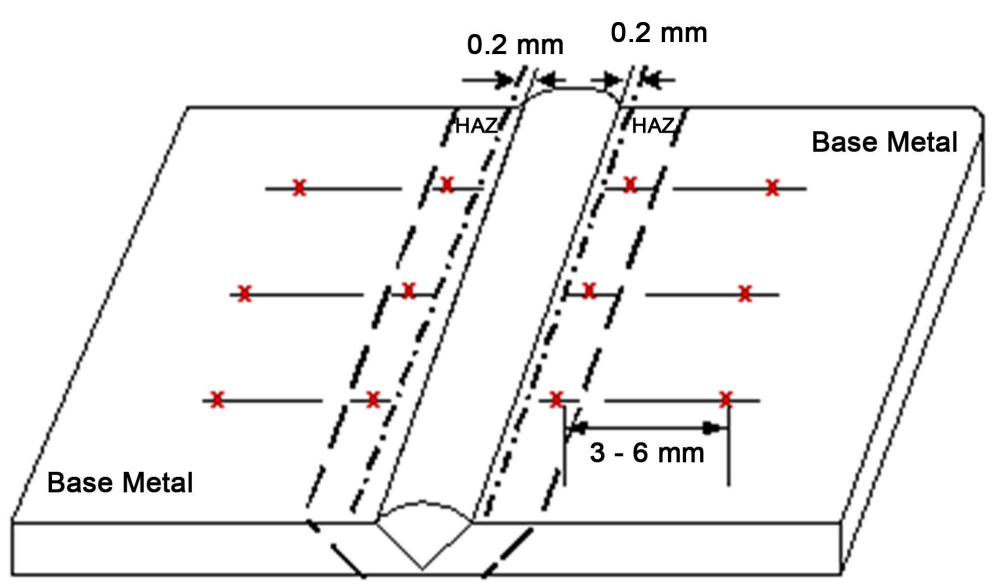

Figure 2. Butt welded joint with the sample locations of hardness testing.

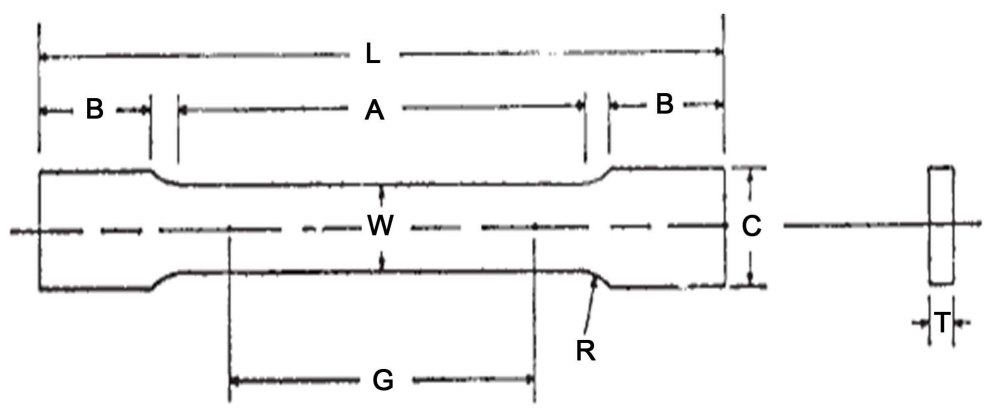

Figure 3. Showing the standard machined for tensile test.

\subsection{Metallographic Test}

Metallographic samples were produced from welds in accordance to ASTM E 23. Grinding, Polishing, etching with $2 \%$ Nital and Metallographic examination of butt and groove welds were performed using optical microscope Olympus PMG3 with magnification $\times 500$.

\section{Results and Discussion}

Figure 4 clearly shows the mechanical properties of the welded steel at fixed Voltage (220 V) and varying Current in Straight Edge preparation welded sample. It was discovered that current adjustment affects the weld bead size, appearance, penetration and strength of the weld. There was a decrease in Hardness and Tensile strength of the welded steel as the current was increased. With increased current, the heat generated increased causing the grain to recrystallized and grow in size. Increased in grain size increases the impact strength (toughness) of the weld but reduction in hardness and tensile strength of weldment and heat affected zone as depicted by the graph. This was in agreement with [4]. Similarly, the amount of heat input from the heat source is given by

$H=\frac{(I \times V) 60}{S}$.

This shows that as the current increases more heat is produce into the weld causing more expansion and contraction between the weldment and the base metal. This increased the residual stresses resulting in lower mechanical properties.

Figure 5 shows the mechanical properties of the welded steel at fixed voltage (220 V) and varying current in the V groove edge welded sample. From this plot, the hardness as well as the tensile strength of the weldment and heat affected zone decreased with an increase in the current. This again is due to increase in heat input into the weldment. The heat persisted for a period of time and is quickly conducted away into the base metal as a result of low power density of the welding process (SMAW) as reported by Sindo [1]. Consequently, the based metal was affected due to high heat input into the weld resulting in grain size increased. This case is also similar 


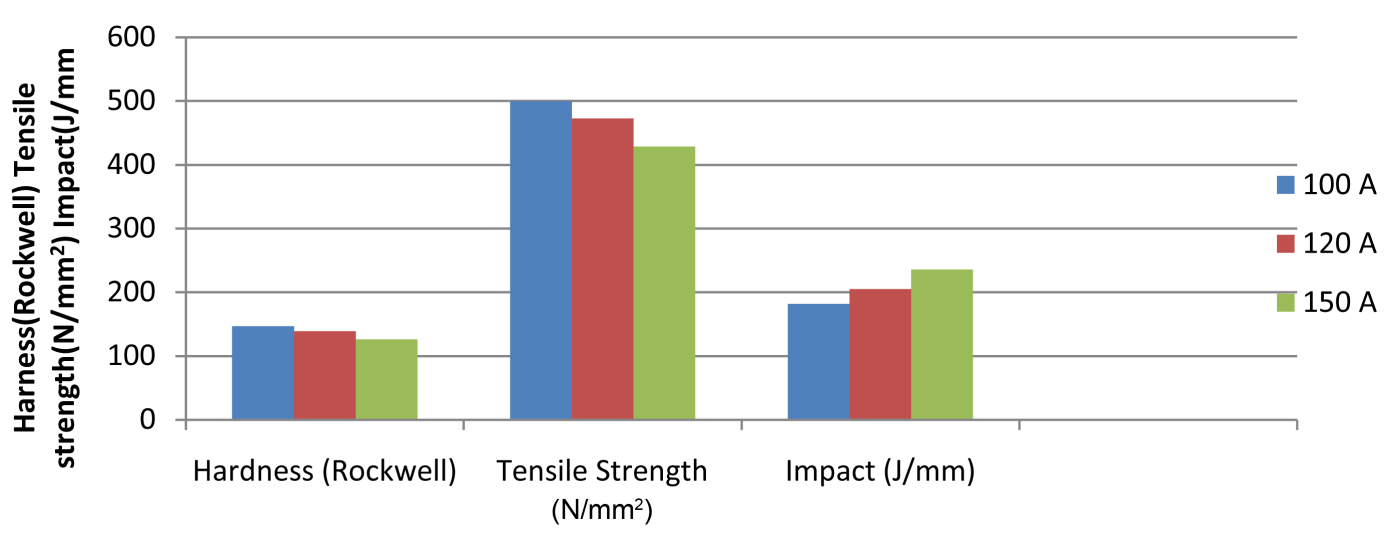

Figure 4. Fixed voltage (220 V) and varying current with straight edge.

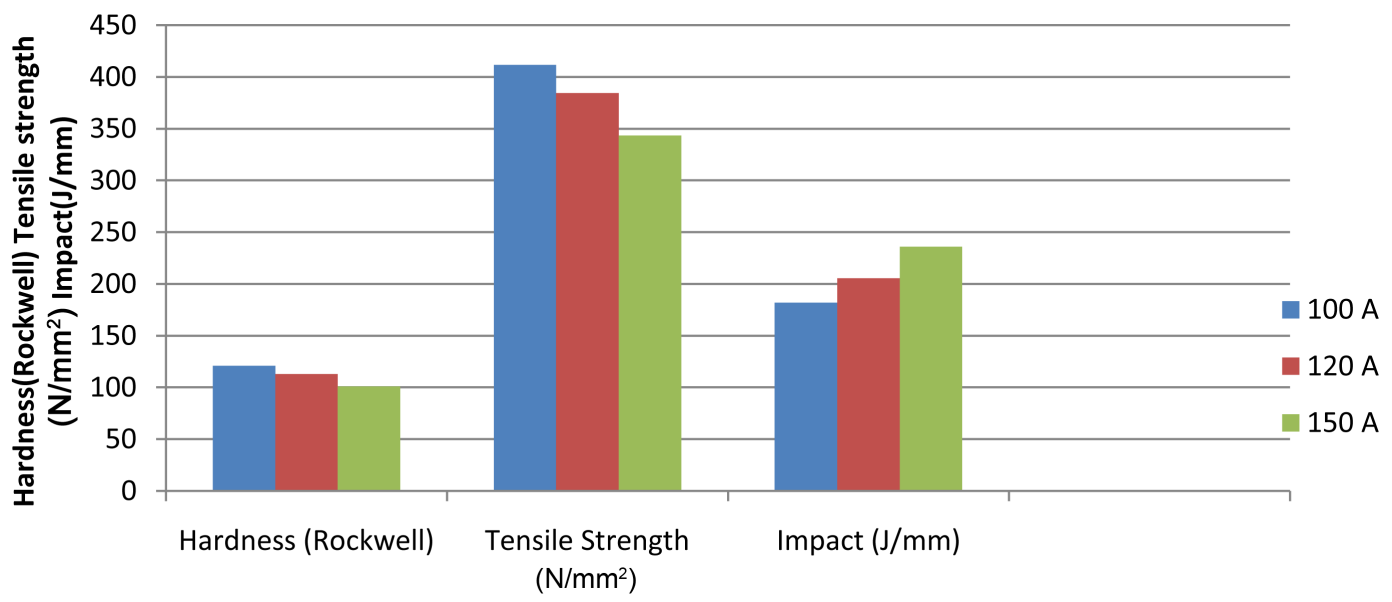

Figure 5. Fixed voltage (220 V) and varying current with V groove edge.

to what happened in Figure 4. The only exception is that Figure 5 is with V-grooved edge with high amount of weldment deposit. This increased the residual stresses in the weldment and heat affected zone. Residual stresses can significantly affect the engineering properties of the material and this has contributed to reduction in strength and hardness of the weldment and heat affected zone as shown in the Figure 5.

In Figure 6, the current was fixed at $100 \mathrm{~A}$ while the voltage was varied from $100 \mathrm{~V}$ to $200 \mathrm{~V}$. The hardness and the tensile strength of the weldment and the heat affected zone reduced as the voltage increases. This is similar to that of Figure 4 and Figure 5 as they all conformed to the mathematical model equation relating heat source and welding parameters (current and voltage) [5] [6]. Besides it is difficult to maintain constant gap between the arc and the weldment in manual welding, and as such the system is self-adjusting such that increase in voltage will not cause significant increase in welding current as reported by Tariq in his lecture 2014 at King Fard University of Petroleum Saudi Arabia.

Figure 7 shows similar phenomenon as that in Figure 6, though the edge preparation differences caused significant changes in the mechanical properties. It was observed in Figure 6 that the tensile strength at $100 \mathrm{~A}$ is about $500 \mathrm{~N} / \mathrm{mm}^{2}$ while the corresponding tensile strength in Figure 7 is about $420 \mathrm{~N} / \mathrm{mm}^{2}$. This is because grove edge accommodates more weld deposit than straight edge and therefore the bonding strength should have been better than that of straight edge. As reported in several literature, the more the weld deposit, the higher the expansion and contractions consequently increase in residual stresses in the weldment and the heat affected zone. Gery et al. [4] noted that, residual stresses can significantly affect the mechanical properties of the weldment. It was also noted that the impact strength is slightly higher in Figure 7 than in Figure 6 with the similar reasons explained above.

Figure 8 and Figure 9 depict fixed current and voltage with different edge preparation. The phenomenon in both figures was almost the same as there were no changes in the heat input into the weldment. However differ- 


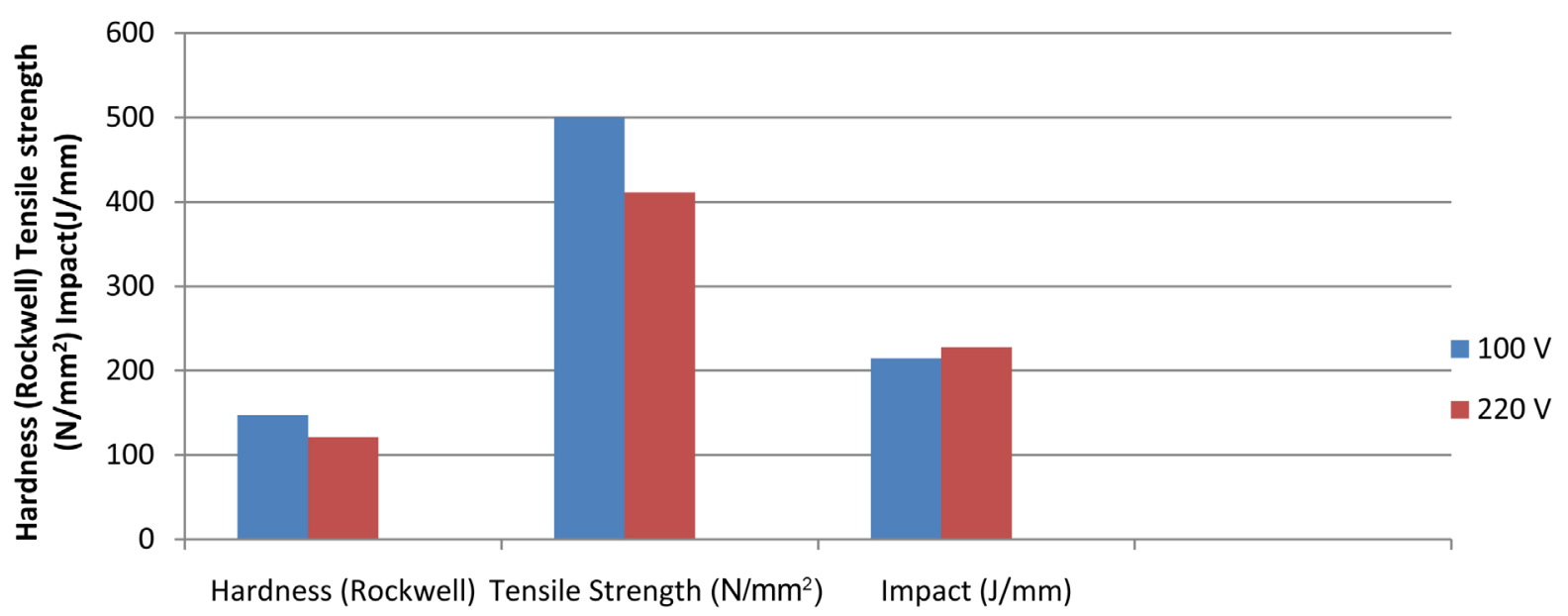

Figure 6. Fixed current (100 A) varying voltage with straight edge.

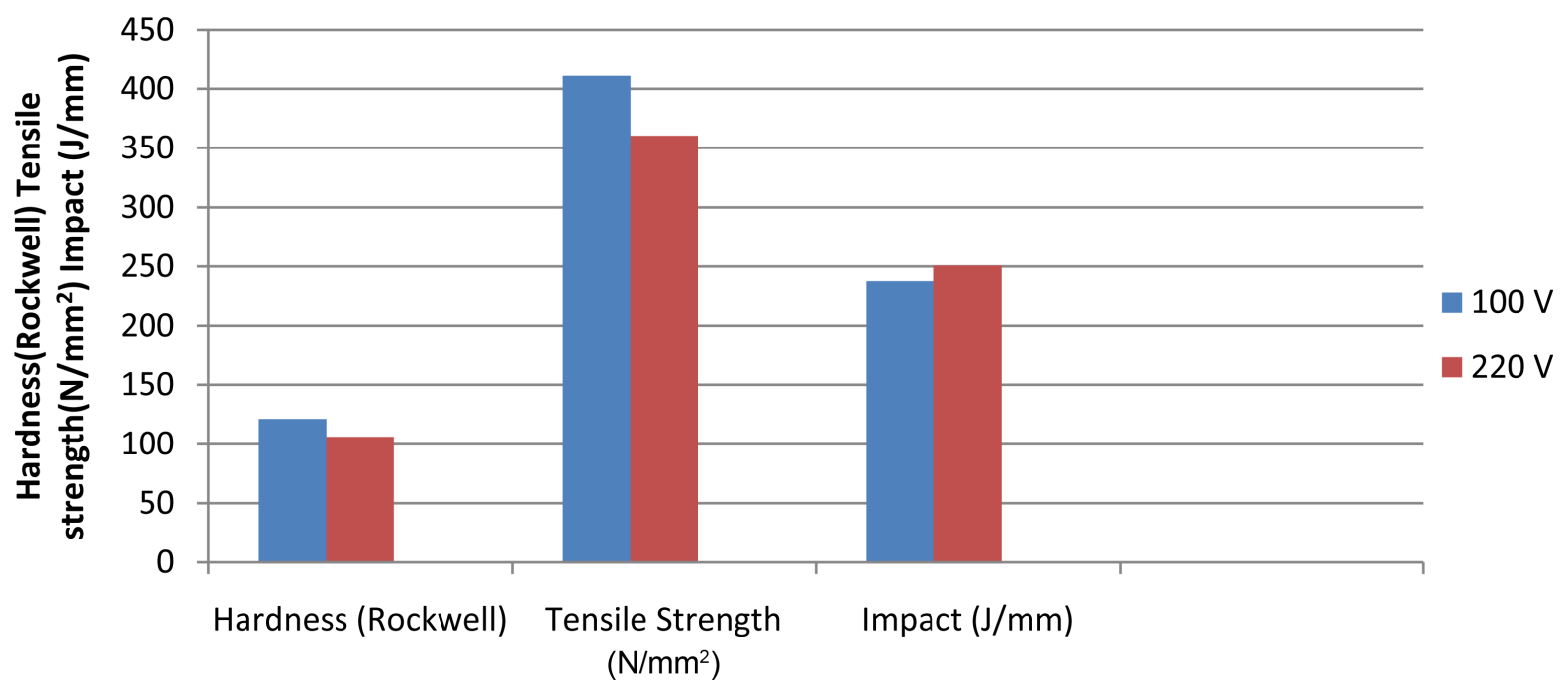

Figure 7. Fixed current $(100 \mathrm{~A})$ varying voltage with $\mathrm{V}$ groove edge.

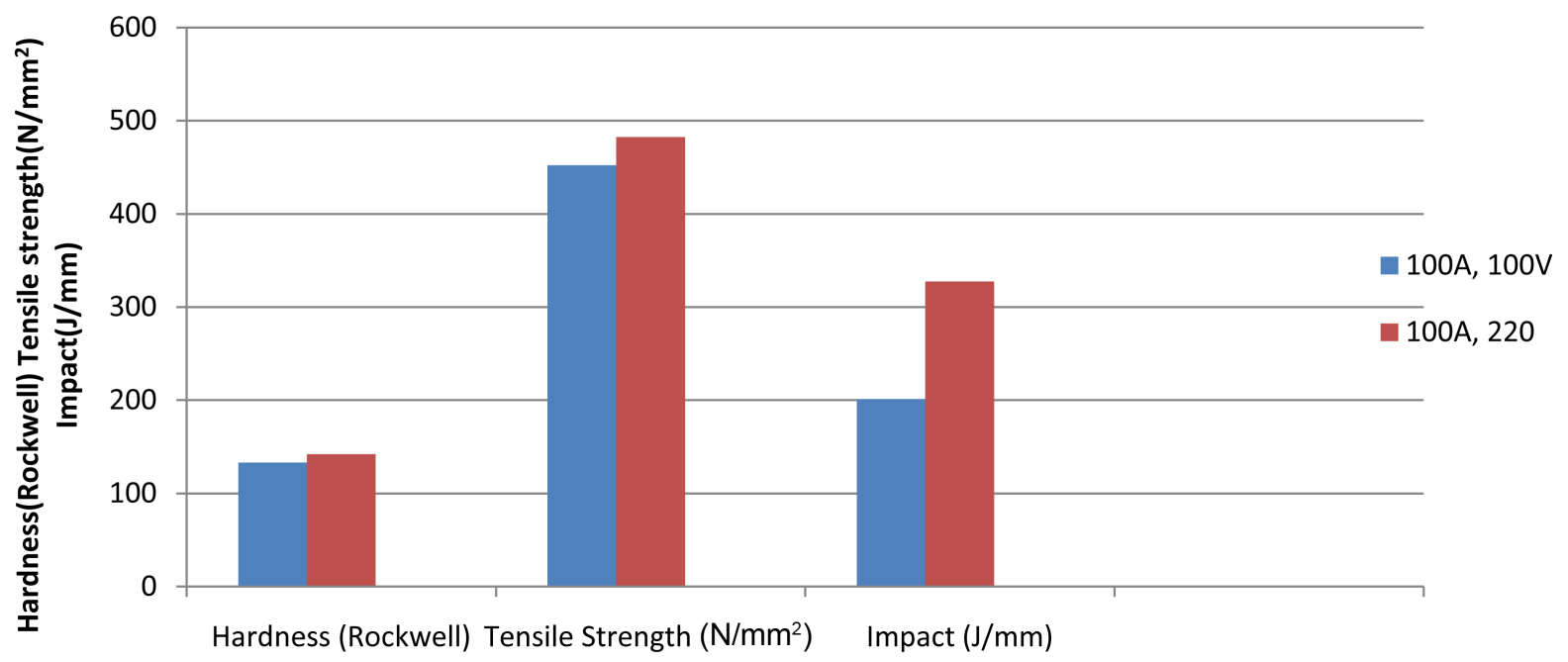

Figure 8. Fixed voltage and current with straight edge preparation. 


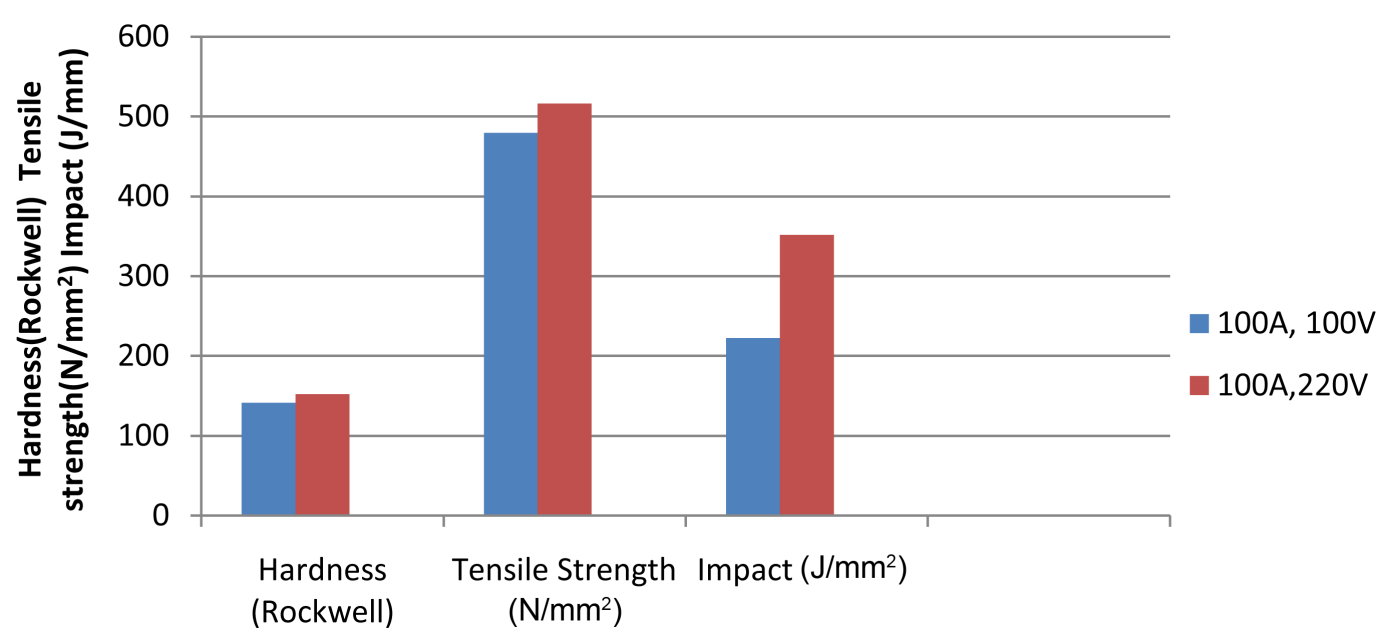

Figure 9. Fixed voltage and current for different edge preparations with $\mathrm{V}$ groove edge.

ences in edge preparations caused slight disparity in the values of the mechanical properties of the two types of preparation under study. Tensile strength is higher in V-grooved edge in Figure 9 than that of straight edge in Figure 8 as the effect of bonding is dominant over the residual stress due to constant heat input into the weldment. Similarly, the impact strength (toughness) also improved slightly in V-grooved edge in the Figure 9 than the corresponding impact in Figure 8.

In Figure 10 and Figure 11, the same scenario is exhibited except that the electrode is of different compositions. It was discovered that the use of different electrode at constant current and voltage did not have any significant changes in the tensile strength and hardness of the welded steel. However there is a noticeable improvement in the impact strength in V-grooved edge in Figure 11 than that of Figure 10.

Figure 12 and Figure 13 shows the mechanical properties of the welded steel at fixed current and voltage for different cooling medium with straight edge and V-grooved edge preparations respectively. The cooled in still air samples has a lower tensile strength, and higher hardness values as shown in Figure 12. This may be due to a slow rate of cooling in draft air than that of still air. Low cooling rate led to grain growth of the welded joint [3] [8] [9]. In Figure 13, it was observed that there was decrease in the hardness value of the sample cooled in the still air, and this can be attributed to decrease in the concentration of hydrogen on the joint surface that caused long time or delay in cooling rate.

Figure 14 shows the comparison of the mechanical properties of samples welded with oxy-acetylene in straight edge and V-Beveled edge preparations. From this figure, the tensile strength and the hardness values of the two types of preparations were totally different. As compared to SMAW, the mechanical properties of OAW as reported in Figure 10 is lower than that of SMAW shown in Figure 4 and Figure 5 respectively. This is due low power density of OAW process.

The results of microstructural examination revealed that all the welded joints contain pearlite and ferrite phases.

Figure 15(a) shows the structure of the Base Metal of the Low Carbon Steel used for this study. The structure consists of about $80 \%$ to $85 \%$ ferrite (white) and about $15 \%$ to $20 \%$ pearlite (black).

It was observed that the grain-refined area exhibits extremely small ferrite grains, clearly indicating that very fine ferrite and pearlite is formed due to the heating and cooling cycles of the SMAW process [7].

Figure 15(a) and Figure 15(b) show the microstructures of the Weld Zone of samples welded at Constant Voltage and varied Current setting, representing a grain-coarsened area, which exhibits predominantly White Ferrite (WF) and pearlite.

Figure 16(a) and Figure 16(b) show the structure of the samples welded at constant current, and varied Voltage setting. It was observed that a large amount of pearlite is present in the ferrite matrix. As the current was increased, the pearlites become finely distributed within the coarse matrix with an increase in the proportion of the ferrite to pearlite.

Figure 17(a) and Figure 17(b) compare the prepared straight edge and the prepared V-grooved edge and it was discovered that ferrite phase were more than pearlite phase in their microstructure which also indicates 


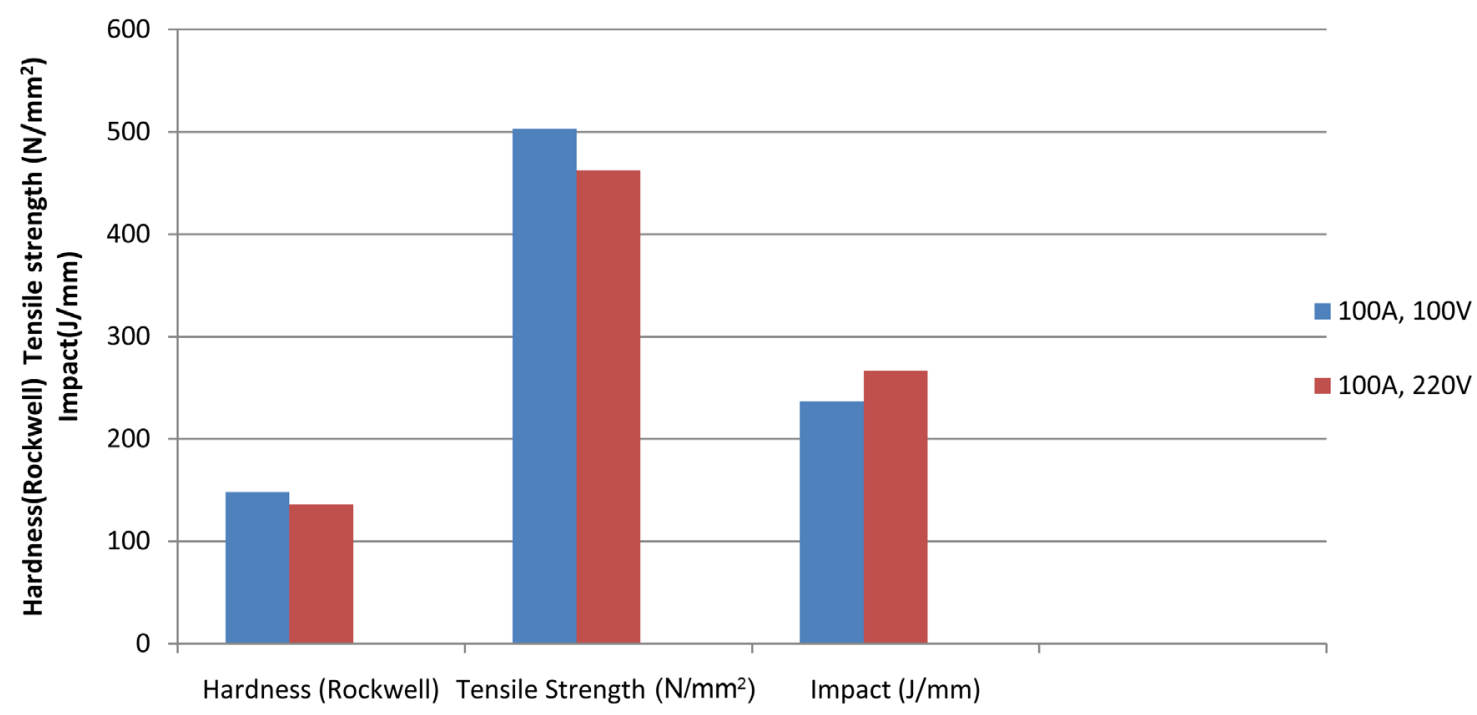

Figure 10. Fixed voltage and current for different types of electrode with straight edge.

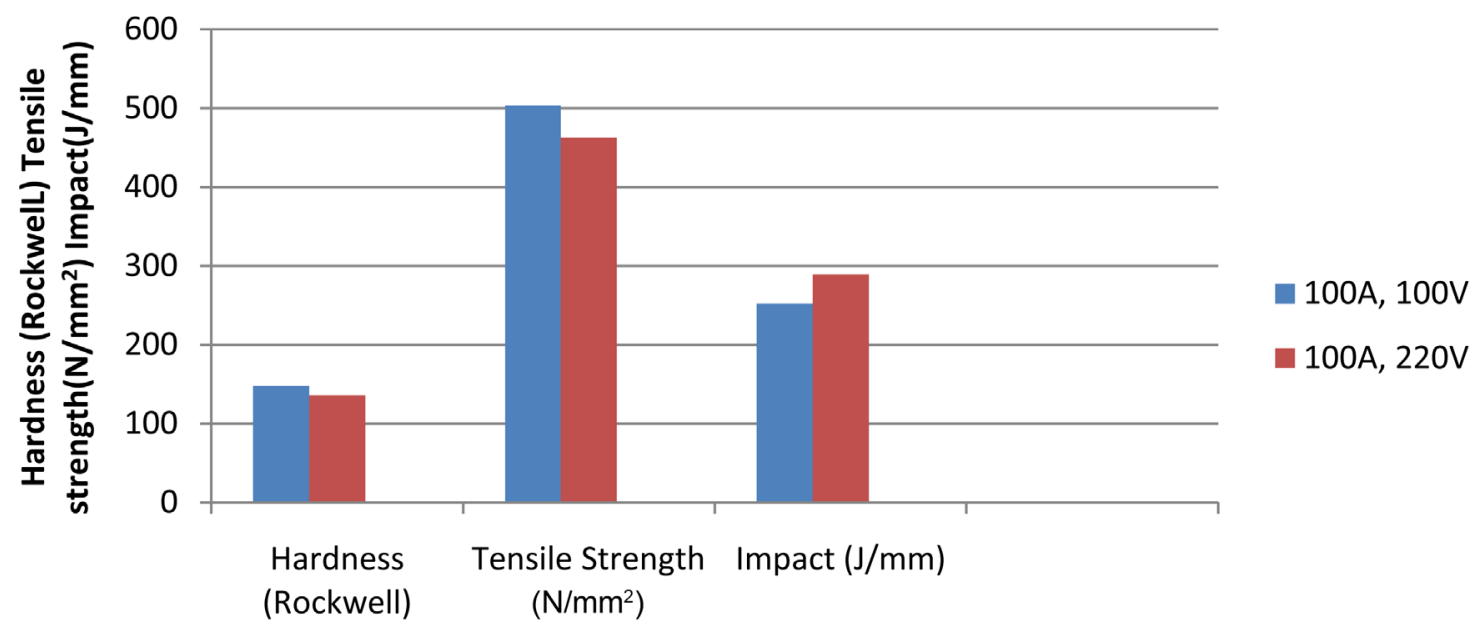

Figure 11. Fixed voltage and current for different types of electrode with $\mathrm{V}$ groove edge.

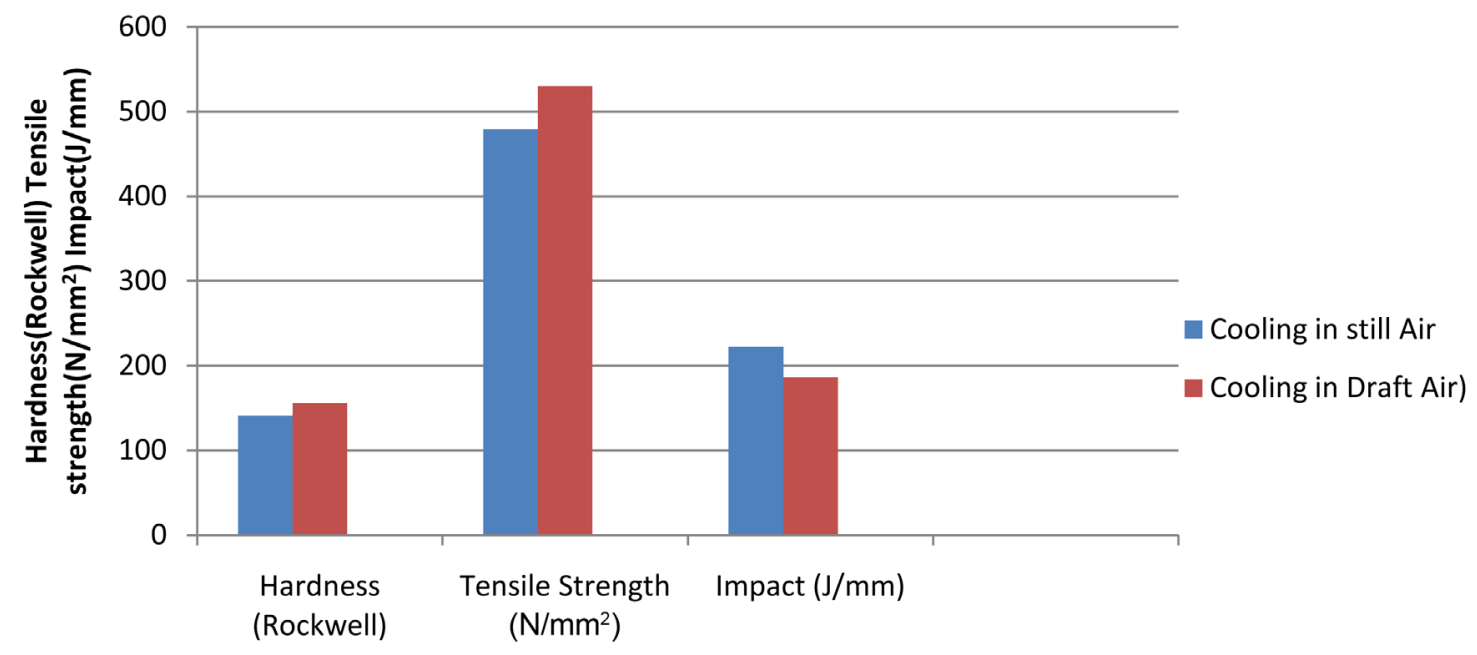

Figure 12. Fixed voltage and current for different cooling medium with straight edge. 


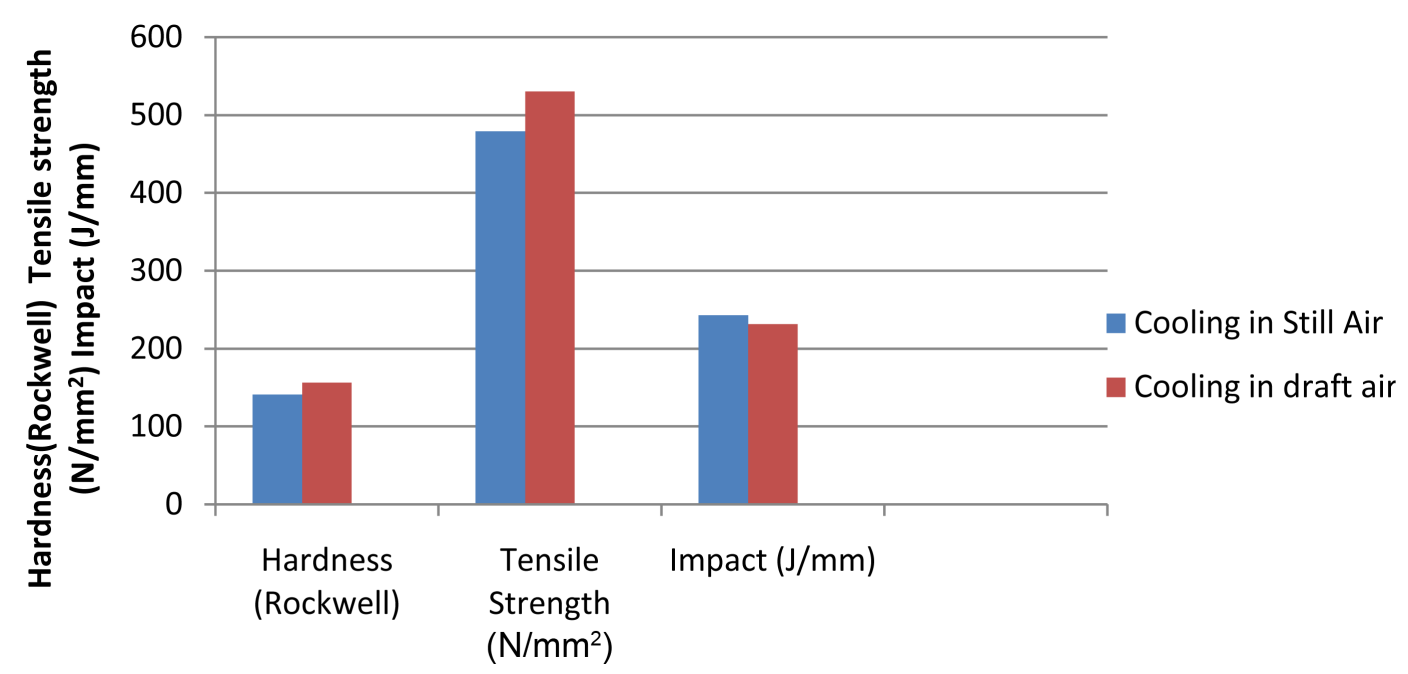

Figure 13. Fixed voltage and current for different cooling medium with V groove edge.

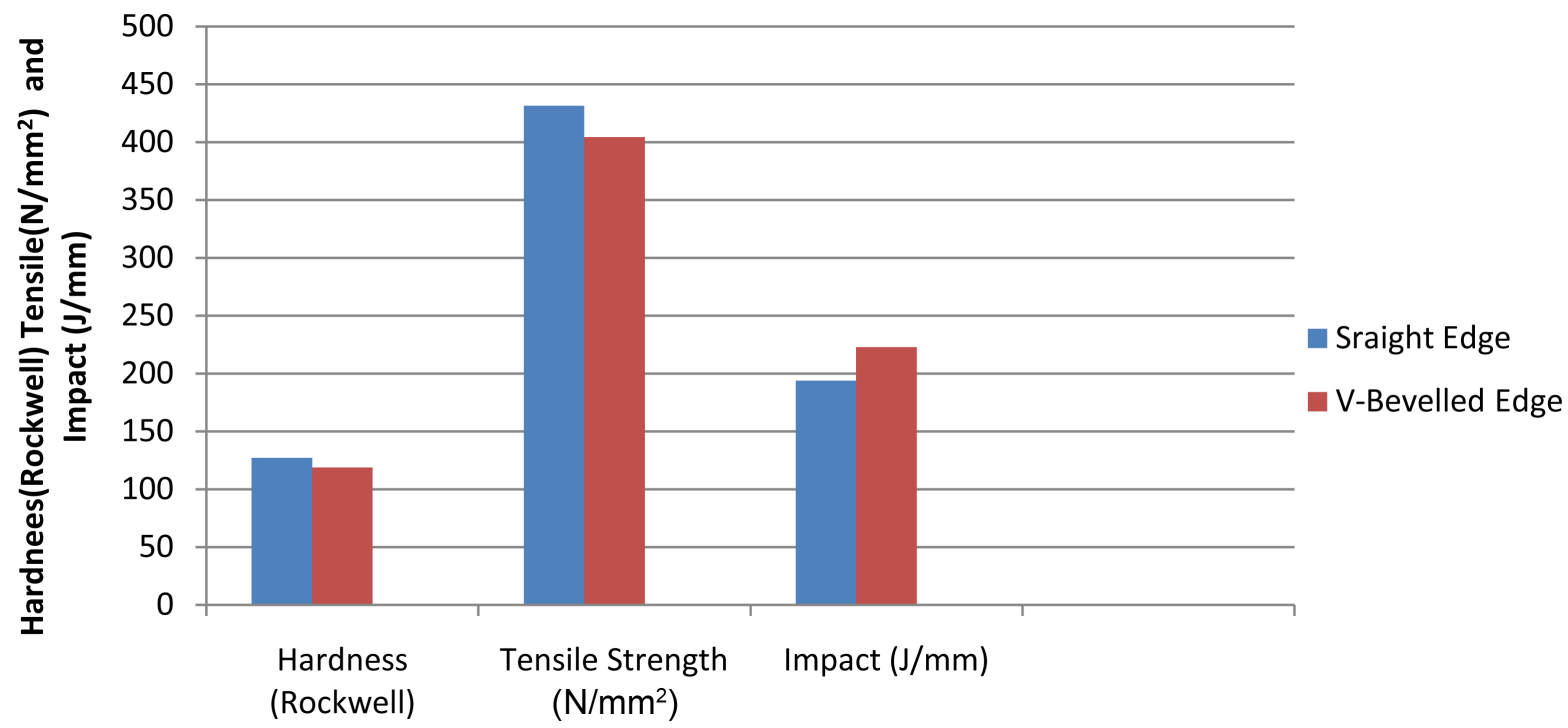

Figure 14. Welding with oxyacetylene flame with straight edge and V-bevelled edge.

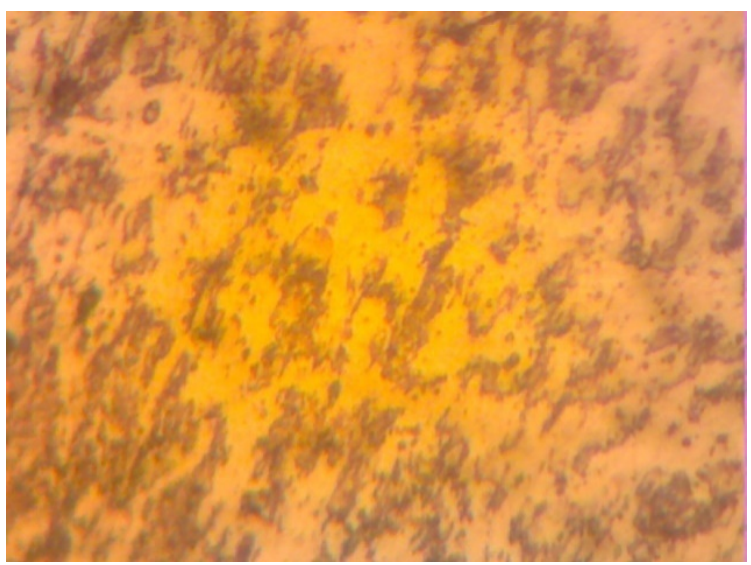

(a)

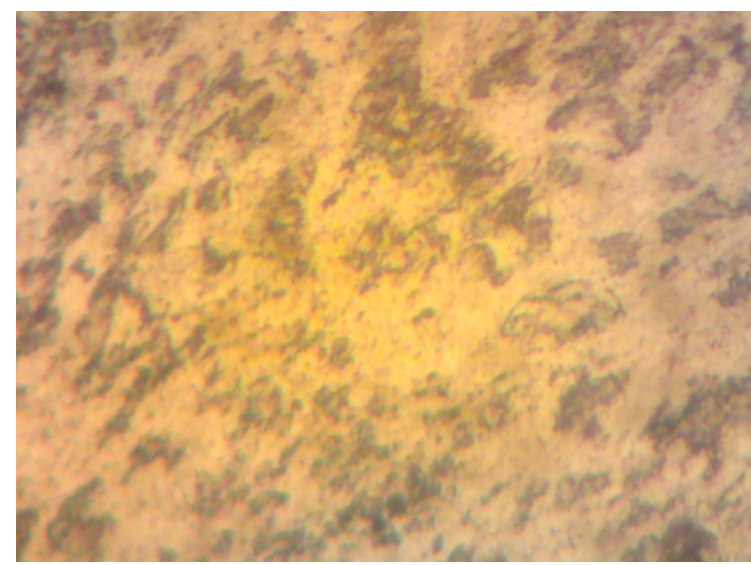

(b)

Figure 15. (a) Fixed voltage (220 V) and varying current; (b) Base metal with straight edge. 


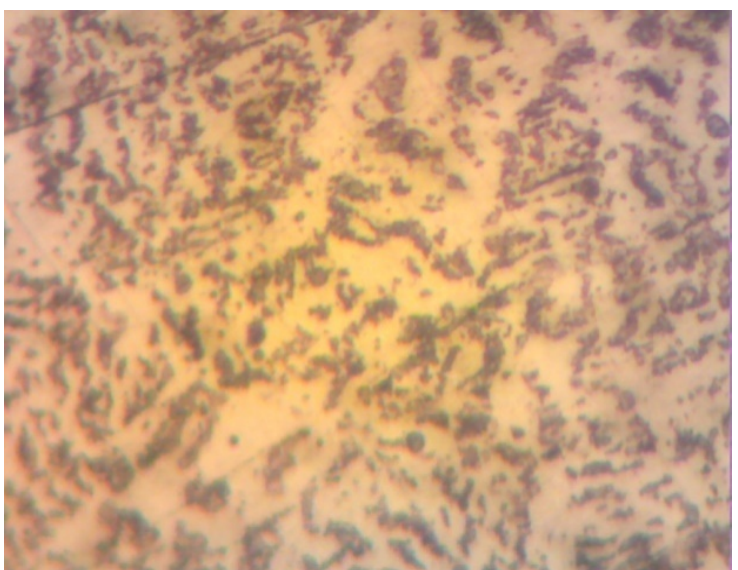

(a)

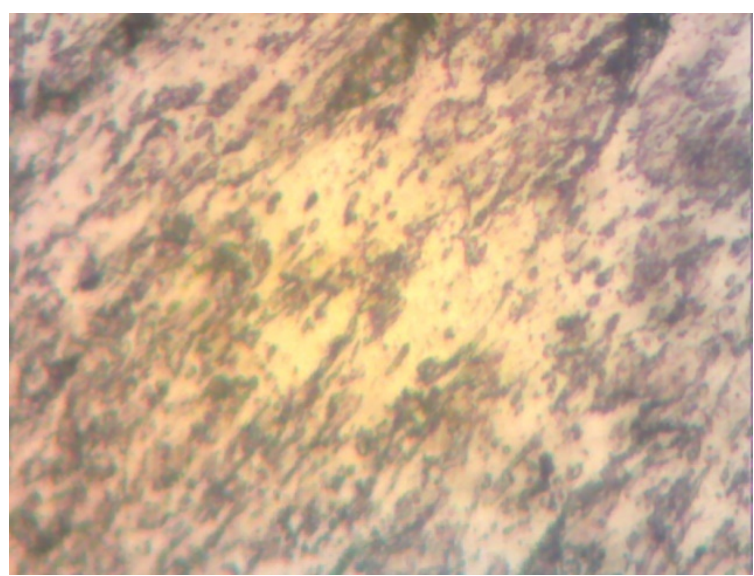

(b)

Figure 16. (a) Fixed current (100 A) varying voltage with straight edge; (b) Fixed voltage (220 V) and varying current with $\mathrm{V}$ groove edge.

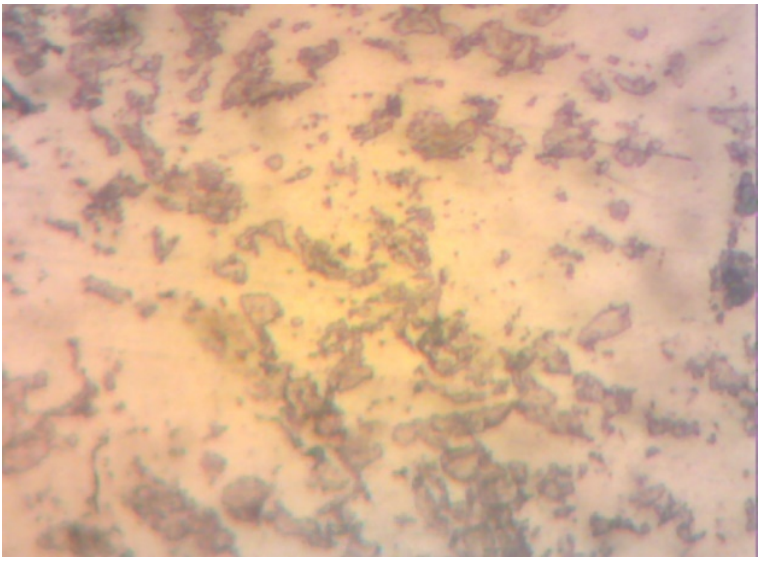

(a)

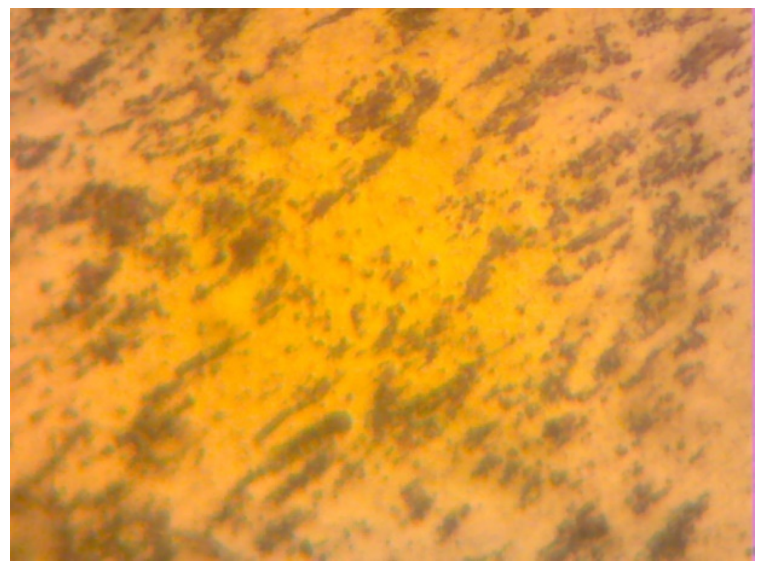

(b)

Figure 17. (a) Fixed voltage and current for edge preparations with straight edge; (b) Fixed current (100 A) and varying voltage with $\mathrm{V}$ groove edge.

higher ductility in Figure 17(a); this is in agreement with the work of [5] [6].

Examination of the Welded Zones in Figure 18(a) and Figure 18(b) also revealed the effect of electrode size on the microstructure. The observed structure is of coarse pearlite in ferrite matrix which became more coarse as the grain size increased. This observation explained the decrease in the hardness and tensile strength values as the heat energy input was increased.

Microstructure for samples welded with fixed voltage and current setting for different cooling medium were shown in Figure 19(a) and Figure 19(b). These plates revealed the effects of cooling rate after welding on the properties of the steel. Cooling in still air produces coarse grain structure of both ferrite and pearlite while cooling in draft air produces fine grain size. This is due to slow cooling of the weldment in still air [3].

Figure 20(a) and Figure 20(b) revealed fine-grain microstructure of weldment of sample with Oxyacetylene welding procedure. Fine grain ferrite and pearlite were observed in this area as compared to the base metal microstructure (Figure 19(a)). However, as observed from Figure 21(a) and Figure 21(b) better grain refinement were noticed than in Figures 20(a) and Figure 20(b). This clearly indicates that during the heating and cooling cycles, a very fine grain structure is formed in the FZ. This area is generally termed as the grain-refined area.

From the observation of the plates, the microstructures with higher concentration of the pearlite phase will have more hardness and strength but with lower ductility. Ferrite crystals were formed as a result of transformation in low-carbon steel upon cooling from the austenitic state. It is not possible to state the relationship mathematically, but since the ferrite grains are nucleated and grow; their size will depend on time and temperature, 


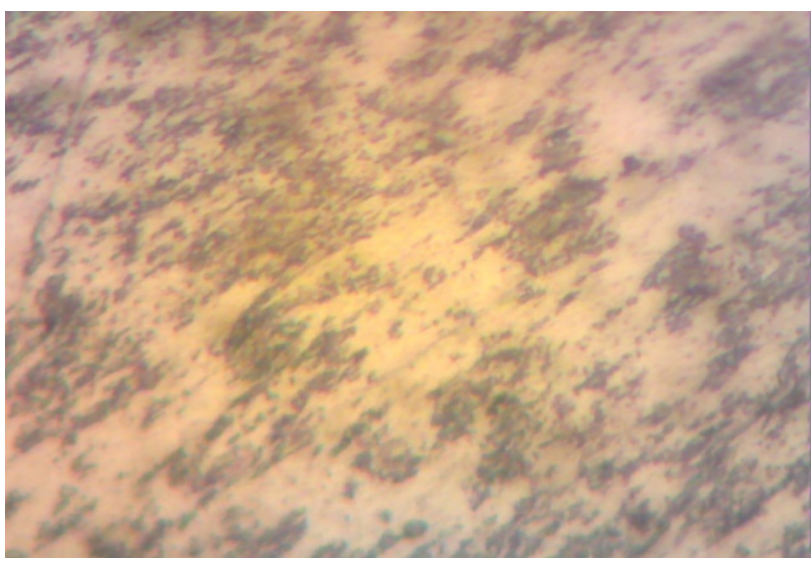

(a)

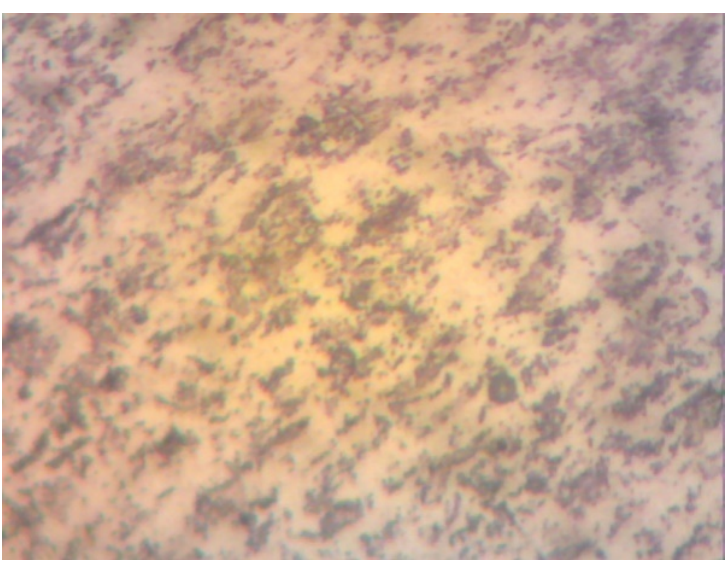

(b)

Figure 18. (a) Fixed voltage and current for different types of electrode with straight edge; (b) Fixed voltage and current for diff edge preparations with $\mathrm{V}$ groove edge.

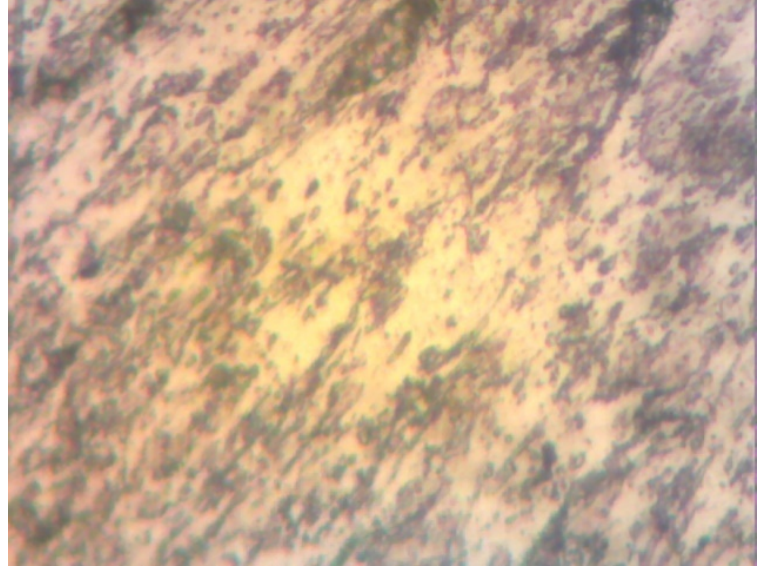

(a)

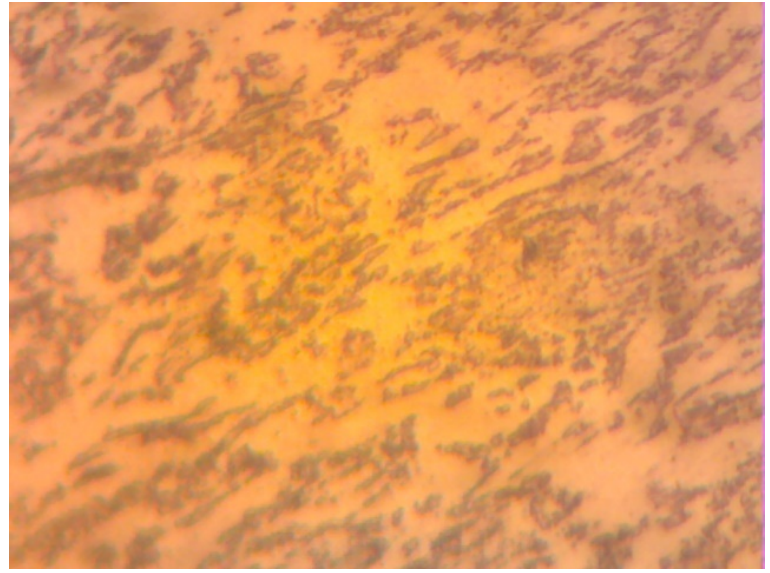

(b)

Figure 19. (a) Fixed voltage and current for medium with straight edge; (b) Fixed voltage and current for different types of electrode with V groove edge.

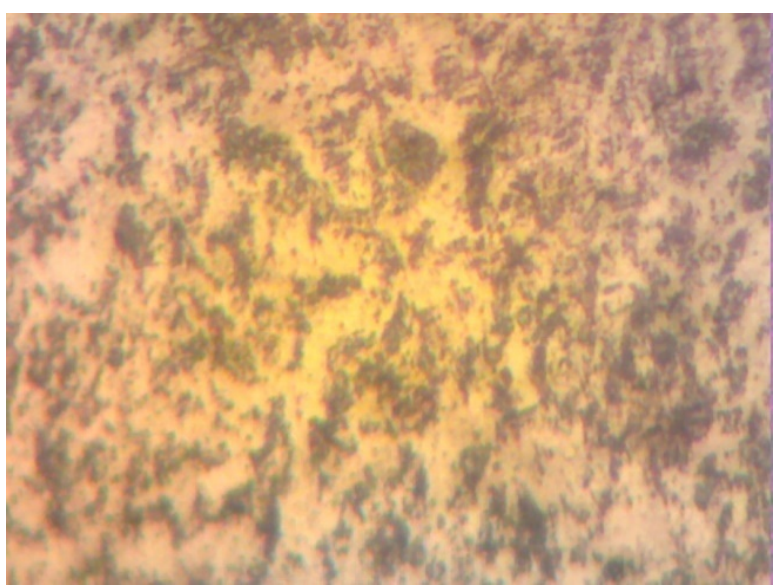

(a)

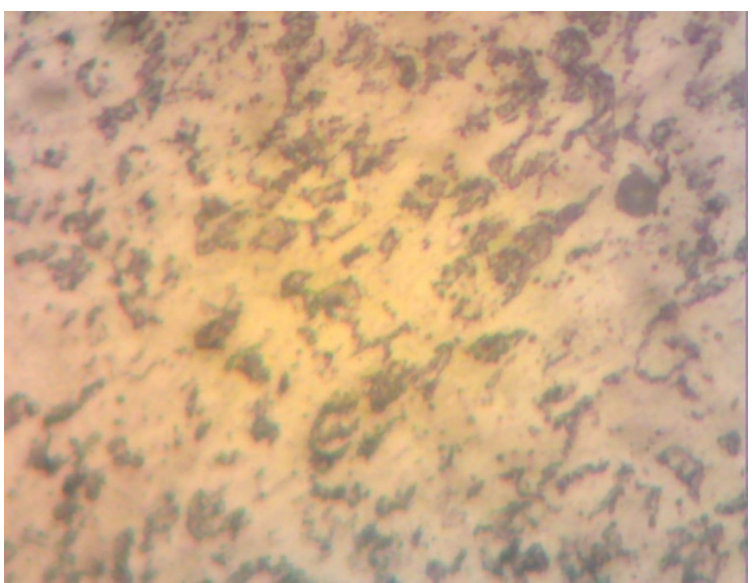

(b)

Figure 20. (a) Welding with oxyacetylene with straight edge; (b) Fixed voltage and current for cooling medium with V groove edge. 


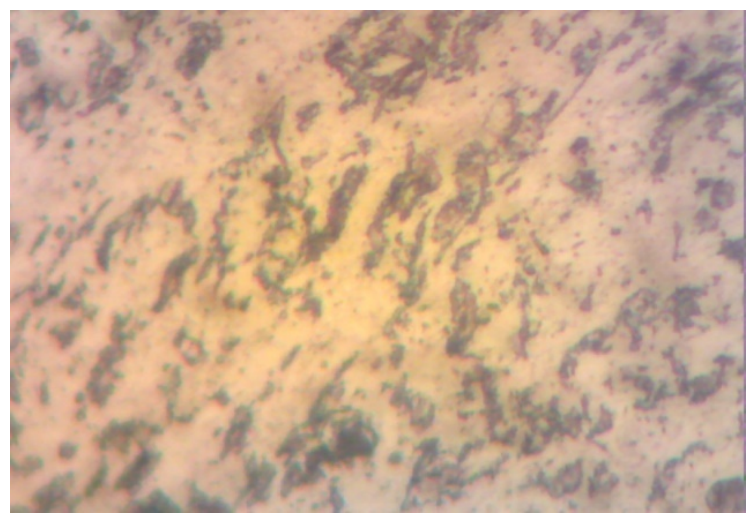

(a)

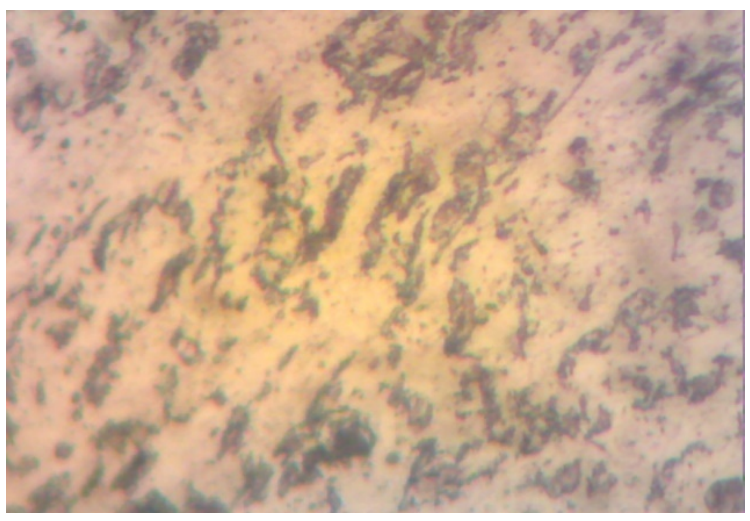

(b)

Figure 21. (a) Fixed voltage (220 V) and varying current with straight edge; (b) Welding with oxyacetylene flame with $\mathrm{V}$ groove edge.

especially on the cooling rate.

\section{Conclusions}

The following conclusion can be drawn from the present study:

- Adequate edge preparation of the weldment enhances the strength of the welded steel. This may be due to the high diffusion of the weld metal which in turn may enhance bonding of the two metal pieces.

- The hardness, tensile and impact strength of the welds are functions of the microstructure; therefore these properties are structural sensitive.

- It was observed that the strength and hardness of the joint increase with the decrease in heat input.

- The toughness increases while the hardness and tensile strength of the joint decrease.

\section{References}

[1] Kou, S. (2003) Welding Metallurgy. 2nd Edition, John Wiley\& Sons, Inc., Hoboken, New Jersey, 17-20.

[2] Puchoicela, J. (1998) Control of Distortion of Wed Steel Structures. Welding Journal, 77, 49-52.

[3] Ueji, R., Fujii, H., Cui, L., Nishiokioka, A., Kunishige, K. and Nogi, K. (2006) Friction Stir Welding of Ultrafine Grained Plain Low-Carbon Steel Formed by the Martensite Process. Materials Science and Engineering: A, 423, 324330. http://dx.doi.org/10.1016/j.msea.2006.02.038

[4] Gery, H., Long, P. and Maropoulos, E. (2005) Effects of Welding Speed, Energy Input and Heat Source Distribution on Temperature Variations in but Joint Welding. Journal of Material Processing Technology, 167, 393-401. http://dx.doi.org/10.1016/j.jmatprotec.2005.06.018

[5] Muthupandi, V., Srinivasan, P., Bala, S.K. and Sundaresan, S. (2003) Effect of Weld Metal Chemistry and Heat Input on the Structure and Properties of Duplex Stainless Steel Welds. Materials Science and Engineering: A, 358, 9-16. http://dx.doi.org/10.1016/S0921-5093(03)00077-7

[6] Yan, J., Goa, M. and Zeng, X. (2010) Study on Microstructure and Mechanical Properties of 304 Stainless Steel Joints by TIG, Laser and Laser-TIG Hybrid Welding. Optics and Lasers in Engineering, 4, 512-517. http://dx.doi.org/10.1016/j.optlaseng.2009.08.009

[7] Monika, K., Bala, M.C., Nanda, P.K. and Prahalada, K.R. (2013) Effect of Heat Input on the Mechanical Properties of MIG Welded Dissimilar Joints. International Journal of Engineering Research \& Technology, 2.

[8] Easterling, K.E. (1998) Modeling the Weld Thermal Cycle and Transformation Behavior in the Heat Affected Zone. In: Cerjak, H. and Easterling, K.E., Eds., Mathematical Modeling of Weld Phenomenon, The Institute of Materials.

[9] Marashi, P., Pouranvari, M., Amirabdollahian, S. and Abedi, G. (2008) Microstructure and Failure Behavior of Dissimilar Metal Spot Welds between Low Carbon Steel, Galvanized and Austenistic Stainless Steels. Materials Science and Engineering: A, 420, 175-180. http://dx.doi.org/10.1016/j.msea.2007.07.007

[10] Lowe, T.C. and Zhu, Y.T. (2003) Commercialization of Nanostructured Metals Produced by Severe Plastic Deformation Processing. Advanced Engineering Materials, 5, 373-378. http://dx.doi.org/10.1016/j.msea.2007.07.007 